\title{
El Clima Escolar Como Elemento Fundamental de la Convivencia en la Escuela
}

\section{The School Climate As A Fundamental Element of School Coexistence}

\author{
Ketty Herrera Mendoza \\ Candidata a Doctor en Ciencias Humanas por la Universidad del Zulia. Magister en Psicología. Docente de la Facultad de Psicología de la Universidad de \\ la Costa CUC, adscrita al grupo de Investigación CES (Cultura, Educación y Sociedad). kherrera10@cuc.edu.co.
}

\section{Reinaldo Rico Ballesteros}

Especialista en enseñanza de la Historia de la Universidad del Atlántico (Barranquilla-Colombia). Licenciado en Ciencias Sociales por la Universidad Simón Bolívar. Docente de la Facultad de Humanidades de la Universidad de la Costa, CUC, adscrito al grupo de investigación GIDIPES. rrico2@cuc.edu.co

\section{Para citar este artículo}

Herrera, K., Rico, R., \& Cortés, O. (2014). El clima escolar como elemento fundamental de la convivencia en la escuela. Escenarios, 12(2), 7-18.

Recibido: Julio 152014

Aceptado: Agosto 202014

\section{RESUMEN}

El clima escolar está definido como la cualidad del ambiente escolar experimentado por los estudiantes, docentes y directivos, que al basarse en la percepción que poseen sobre el contexto escolar, determina sus conductas (Hoy y Miskel, 1996). Desde la investigación educativa, indagar acerca de los procesos de interacción escolar implica involucrarnos en una esfera subjetiva, caracterizada por la búsqueda de elementos que le dan sentido a la realidad vivida en la cotidianidad de la escuela; razón por la que se realiza la presente investigación, que tuvo como objetivo fundamental caracterizar el clima escolar en la Institución Educativa Distrital (IED), como base para el mejoramiento de la convivencia en las escuelas del Distrito de Barranquilla. Para lograr tal fin se diseñó una metodología de tipo descriptivo-analítico de corte transversal, en la que se tomó una muestra de 152 estudiantes e igual número de docentes y directivos de las instituciones educativas oficiales de la ciudad de Barranquilla, a quienes se les aplicó una adaptación del "Protocolo para la Medición de la convivencia en los Centros Educativos No Universitarios de la Comunidad Autónoma de Aragón” (Gómez, Puyal, Sanz, Elboj y Sanagustin, 2006). Los resultados indican que se percibe un clima escolar bueno (68,7\% estudiantes y $70 \%$ docentes y directivos). Un $97.1 \%$ de los estudiantes considera que lo que los motiva a ir a la escuela es su deseo de aprender y que se trata de un escenario para obtener un mejor futuro (86\%). El 78\% de la muestra considera que la escuela le proporciona tranquilidad y un $78 \%$ la concibe como un lugar para compartir entre pares. La información cualitativa obtenida de observaciones y entrevistas indica que la interacción presenta escenarios de irrespeto que conducen a la generación de conflicto escolar, tales como irrespeto, enfrentamiento entre pares, egoísmo, ofensas verbales, apodos, burlas y agresiones físicas, hurto, extorsión, amenaza y daño en el bien ajeno.

Palabras Clave: Convivencia Escolar, Clima Escolar, Educación, Violencia Escolar.

\begin{abstract}
The school climate is defined as the quality of the school environment experienced by the students, teachers and administrators, which based on the perception that they have on the school context, determines their behavior (Today and Miskel, 1996). From the educational research, inquire as to the processes of school interaction involves getting involved in a subjective area, characterized by the search for elements that give meaning to the reality experienced in the everyday life of the school; why is this research, which took as its fundamental objective characterize the climate in the school district Educational Institution (FDI), as a basis for improving the coexistence in the schools of the district of Barranquilla. For this purpose a methodology was designed for descriptive-analytical of sectional, which took a sample of 152 students and an equal number of teachers and administrators of the formal educational institutions of the city of Barranquilla, was applied to them an adaptation of the "Protocol for the measurement of coexistence in the non-university educational centers of the
\end{abstract}


Autonomous Community of Aragon" (Gomez, Puyal, Sanz, Elboj and Sanagustin, 2006). The results indicate that there is a good school climate ( $68.7 \%$ students and $70 \%$ teachers and executives). A 97.1 per cent of the students considered that what motivates them to go to the school is its desire to learn and that it is a scenario for a better future (86 \%). The $78 \%$ of the sample considered that the school gives you peace of mind and a $78 \%$ conceives it as a place to share between pairs. The qualitative data obtained from observations and interviews indicate that the interaction presents scenarios of disrespect that lead to the generation of school conflict, such as disrespect, confrontation between pairs, selfishness, verbal offenses, nicknames, teasing and physical assaults, theft, extortion, threats and damage to the good of others.

Key words: School Coexistence, School Climate, Education, School Violence

\section{Introducción}

Hablar de calidad educativa en la actualidad, implica no sólo revisar y mantener actualizado el modelo pedagógico y los contenidos curriculares empleados las instituciones educativas, sino también incluir elementos que favorezcan una buena convivencia escolar y que sin duda posibiliten una formación integral de los estudiantes.

Las acciones llevadas a cabo en las escuelas en materia de convivencia escolar implican la interacción de todos los elementos de la comunidad educativa: a) El currículo, con toda una gama de contenidos, metodologías, estrategias pedagógicas, mediaciones personales y sociales (ética y valores); b) La relación entre la familia y la escuela, como soporte a los procesos educativos y apoyo primario en el fortalecimiento del trabajo en la escuela; y c) La influencia de los medios de comunicación y del entorno socio-económico y cultural en el que se encuentran involucrados los estudiantes. El funcionamiento adecuado de los distintos factores de la convivencia escolar es lo que configura el clima escolar, al tratarse de un escenario psicológico en el que cada miembro de la comunidad escolar concibe la escuela y sus acciones.

\section{Clima Escolar}

El desarrollo de la variable "Clima escolar" tiene como antecedente el concepto de "Clima Organizacional", que desde la psicología social, toma elementos de la teoría general de sistemas en su afán por comprender el comportamiento de los individuos en los escenarios laborales y se extiende al estudio de otro tipo de fe- nómenos sociales desde una concepción integradora (Rodríguez, 2004). Específicamente, para la definición de "Clima escolar" confluyen diversas teorías psicosociales, relacionadas con la interacción entre necesidades y motivaciones de las personas con los factores de tipo social. Tales teorías tienen sus inicios en los postulados de Kurt Lewin (1890 - 1947) analizados a partir de Lacouture (1996), basados en el modelo interaccionista, y que posteriormente se expandieron en Estados Unidos, con los aportes de Murray (Cornejo y Redondo, 2001).

Basados en los distintos enfoques que han abordado el tema, el clima escolar puede ser definido como "El conjunto de características psicosociales de un centro educativo, determinadas por aquellos factores o elementos estructurales, personales y funcionales de la institución que, integrados en un proceso dinámico específico, confieren un peculiar estilo a dicho centro, condicionante, a la vez de los distintos procesos educativos" (Cere, 1993:30). Sin embargo, vale la pena aclarar, luego de analizar la definición de Cere, es que sin duda el individuo juega un papel fundamental, puesto que es él quien otorga a la institución educativa las características psicosociales a las que se hace referencia. En este sentido, el clima escolar será definido teniendo en cuenta las percepciones de los miembros del entorno educativo en cuanto a las relaciones interpersonales establecidas entre ellos.

En términos generales, el clima escolar incluye, tanto a la percepción que los niños y jóvenes tienen sobre su contexto escolar, como la que tienen los profesores acerca de su entorno laboral (Arón \& Milicic, 2000). Dicho en otros términos, el clima escolar hace refe- 
rencia a los procesos de interacción que se generan entre diversos actores en un contexto y momento determinado, en el que se presenta un intercambio de estímulos, de respuestas, de compartir las diferencias o consentir las mismas de acuerdo a su nivel social y estado psicológico.

En el mismo orden de ideas, Valdés, Treviño, Castro, Carrillo, Bogoya, Costilla y Acevedo, (2008:53), definen elr clima escolar, en el Primer reporte de resultados y Segundo estudio regional comparativo y explicativo sobre los aprendizajes de los estudiantes de América Latina y el Caribe, bajo los siguientes términos:

El grado en el cual el estudiante se siente a gusto en la escuela y en el aula de clases, con base en los sentimientos que despiertan diferentes situaciones del contex to educativo relacionados con sus compañerosy docentes, tiene en cuenta aspectos relacionados con el grado de tranquilidad que siente el estudiante cuando se encuentra en la escuela, el grado de pertenencia a la institución y la relación con sus compañeros.

Bajo dichas pautas, el clima escolar desde el punto de vista conceptual presenta una diversidad de aproximaciones, al decir de Hoy y Miskel (1996:141) lo definen como «una cualidad relativamente estable del ambiente escolar que es experimentado por los participantes, que afecta a sus conductas y que está basado en las percepciones colectivas de las conductas escolares».

De acuerdo con Arón y Milicic (1999), los climas escolares pueden clasificarse en climas nutritivos y tóxicos.

Los climas nutritivos, ofrecen a las personas la sensación de motivación a participar en todas las actividades que se establecen en la escuela, poseen disposición y entusiasmo por aprender y en general aquellas actividades en donde se generan procesos de interacción que favorecen una convivencia positiva. En los climas escolares positivos existe interés por el aprendizaje continuo a nivel académico y social, hay respeto entre los profesores y alumnos, confianza, preocupación por las necesidades de los miembros de la institución, posibilidad de cambio y desarrollo, alta moral, cohesión entre todos y posibilidad de que todos puedan involucrarse en las decisiones de la escuela, mediante el aporte de sus ideas. Asimismo, el clima escolar nutritivo permite un ambiente físico adecuado para las actividades escolares cotidianas, reconocimiento y valoración de críticas y castigos y realización de actividades divertidas, entretenidas y variadas, lo cual genera condiciones que garantizan el mejoramiento de habilidades tanto académicas como sociales en los miembros de la comunidad educativa (Arón y Milicic, 1999).

Por el contrario, los climas tóxicos, se caracterizan por evidenciar una convivencia negativa, interacciones que se derivan en conflictos no favorables para los procesos de enseñanza-aprendizaje ni de interacción entre los miembros de la comunidad educativa. Específicamente, los ambientes negativos obstaculizan el desarrollo de los estudiantes puesto que generan en ellos irritación, estrés, falta de interés y motivación por desarrollar habilidades académicas y sociales, sensación de agotamiento físico y desesperanza; mientras que en los docentes ocasiona un desviamiento de la atención, motivación y compromiso para contribuir a mejorar la visión de futuro de la escuela. Adicionalmente, el clima tóxico puede conducir a que los estudiantes manifiesten apatía por asistir a la escuela y participar en las actividades realizadas en ella, percepción ampliada en los aspectos negativos e interacciones que inevitablemente, en la mayoría de los casos terminan en conflictos (Arón y Milicic, 1999; Ascorra, Arias y Graff, 2003).

El estudio de clima escolar en Colombia es relativamente reciente, incursiones desde la psicología y la medicina han permeado este quehacer y ahora empieza a nutrirse con las disposiciones e indicadores que se desprenden de la guía 34 que expide el Ministerio de Educación Nacional en Colombia. Ello implica, en primera instancia que esta variable compleja y con una diversidad de aristas, se encontraba marginada por la misma acción de cultura investigativa, máxime que uno de los elementos implícitos en el clima escolar, sin duda es la violencia que al interior de las escuelas se generan como consecuencia de los antagonismos y la forma de resolución de los mismos por los actores implicados; la existencia de este tipo de hechos de- 
jaba a la violencia de pares o violencia interpersonal con menor atención interpretativa, en razón a que el núcleo de las grandes investigaciones se concentraba en la violencia política (Chaux, 2010).

Dicho en otros términos, la investigación sobre el quehacer del clima escolar es de relativa novedad en Colombia. Al decir de Erazo (2010) en Colombia, la experiencia sobre el problema de violencia escolar, asociada a la connotación de clima escolar, se inicia en la década de los años 80, con los trabajos de Parra S., Araceli de Tesanos y Camargo M (Peñaranda, 2006); estos productos van constituyendo tendencias investigativas respecto al tema; ahora bien, la producción bebe de otras latitudes tal como lo expone Forero-Londoño (2011) al sostener que entre los estudios empíricos por ejemplo Xesús Rodríguez-Jares (2004), indica la posibilidad de la resolución positiva del conflicto cuando se logre un cambio en el medio social. En ese orden de ideas, el autor en mención también afirma que en la producción de Blaya, Debarbieux, Del Rey-Alamillo y Ortega-Ruiz (2006); y por lo tanto, enfatizan en la violencia interpersonal entre escolares.

\section{Referentes para una aprehensión del clima Escolar en la Institución educativa Distrital (IED) de Barranquilla.}

Luego de más de una década de la implementación en la estructura curricular de las competencias ciudadanas en Colombia, aún queda mucho por hacer en torno a la prevención y mitigación de los conflictos escolares. Si bien el estudio del clima escolar y con él su expresión manifestada en la violencia, se percibe en la mayoría de escuelas públicas y en menor grado en las privadas de la ciudad de Barranquilla, suele divulgarse con mucha frecuencia la diversidad de manifestaciones que presenta el conflicto escolar.

En la actualidad, el análisis del panorama de violencia del país y la ciudad en general, de una u otra forma tiene sus repercusiones en el comportamiento de los individuos en la escuela. Por tal razón, se afirma que los hechos acaecidos en la escuela, son consecuencia de los fenómenos sociales, de los cambios en la misma sociedad, de las etapas evolutivas del Ser Humano. Sin embargo, se ha iniciado todo un proceso para develar esa connotación de estoicismo; la misma empieza a resquebrajarse por el ejercicio de inventario sobre la organización educativa, la organización curricular, la perspectiva de las competencias ciudadanas, la configuración de propuestas axiológicas, la incorporación de otras instituciones sociales a la labor del escuela; entre otras, que tienen como objetivo prevenir y mitigar los conflictos escolares y su manifestación bárbara del uso de la violencia para dirimir los conflictos.

La importancia crucial de todo ello es lo que acontece en el aula; es decir, hay un vuelco en la búsqueda de los síntomas que faciliten un clima escolar favorable, para lo cual ha de abordarse todo hecho que afecte o degrade la convivencia escolar. Como complemento al ejercicio realizado en las escuelas, el desarrollo de políticas públicas ha permitido una atención menos apasionada y más crítica sobre el objeto de investigación en los centros educativos de Barranquilla. (Hoyos, Romero, Valega y Molinares. 2009; Hoyos, 2005; Torres y Páez, 2012; Hoyos, Llanos \& Valega, 2012; Plan de Desarrollo Distrital 2012-2015, Plan Nacional Decenal de Educación 2006-2016). De la misma manera, no se puede desconocer el trabajo que vienen realizando universidades locales al ofertar líneas de investigación en convivencia escolar, conflicto y comunicación, convivencia ciudadana, cultura ciudadana, competencias ciudadanas y competencias afectivas.

Para el estudio del clima escolar en la ciudad de Barranquilla, es necesario, en primera instancia, tener claridad sobre algunos elementos sociodemográficos, que sirven como referentes contextuales para la comprensión de comportamientos dentro del aula de clases. En primera instancia, vale la pena indicar que se trata de un Distrito Especial, industrial y portuario, que cuenta con 1.193.952 habitantes, según proyecciones del Departamento Administrativo Nacional de Estadística (DANE). Se divide en cinco localidades: Murillo Suroccidente, MurilloSuroriente, Norte Centro Histórico; Metropolitana y Riomar.

En ese espacio de $154 \mathrm{~km} 2$, la pobreza se concentra en las localidades Metropolitana, Suroriente y Suroccidente, pero con mayor énfasis en el Suroccidente. El mayor porcentaje de analfabetas lo tienen las localida- 
Tabla 1. Características de las localidades de Barranquilla

\begin{tabular}{|l|c|c|c|c|}
\hline \multicolumn{2}{|c|}{ Porcentaje de población por debajo de: } & \multicolumn{2}{c|}{ Porcentaje de: } \\
\hline \multicolumn{1}{|c|}{ Localidad/municipio } & Línea de pobreza & Línea de indigencia & Analfabetos & Afrocolombianos \\
\hline Metropolitana & 49,6 & 15,1 & 10,7 & 17,0 \\
\hline Norte Centro Histórico & 12,8 & 4,0 & 4,2 & 5,8 \\
\hline Riomar & 22,6 & 5,8 & 5,2 & 7,3 \\
\hline Suroccidente & 61,2 & 20,1 & 9,5 & 13,8 \\
\hline Suroriente & 45,1 & 18,5 & 7,2 & 9,9 \\
\hline
\end{tabular}

Fuente: Cepeda, 2013.

des Metropolitana y Soledad, seguidos de la localidad Suroccidente. La localidad que mayor porcentaje de afrocolombianos tiene es la Metropolitana.

Paralelo a la pobreza, indigencia y analfabetismo, la ciudad de Barranquilla está caracterizada por un alto índice de pandillismo en la población masculina y femenina con edades comprendidas entre los 12 a 26 años.

Tabla 2. Número de pandillas por localidad de Barranquilla.

\begin{tabular}{|l|c|}
\hline \multicolumn{2}{|c|}{ Número de pandillas por Localidad } \\
\hline Localidad & $\begin{array}{c}\text { Número de pandillas } \\
\text { identificadas }\end{array}$ \\
\hline Metropolitana & 33 \\
\hline Norte-Centro Histórico & 1 \\
\hline Riomar & 9 \\
\hline Suroccidente & 26 \\
\hline Sororiente & 22 \\
\hline Área Conurbada -Soledad & 4 \\
\hline $\mathbf{N}^{\circ}$. Total de Pandillas & $\mathbf{9 5}$ \\
\hline
\end{tabular}

Fuente: Hermida, Valencia y Madariaga, 2013.

Hermida, Valencia y Madariaga, (2013), indican que las pandillas son una población expuesta a altos índices de violencia, con participación directa e indirecta de acciones que tienen que ver con homicidios, atracos y riñas. De la muestra de 3000 jóvenes, que tomaron para realizar el proyecto de investigación "Intervención psicosocial a jóvenes en situación de riesgo pertenecientes a pandillas en el distrito de Barranquilla”, un
40\% declaró que se encontraba estudiando en el momento de la encuesta, de la misma forma que un $79 \%$ afirmó haber asistido o es estudiante del sector oficial y un $21 \%$ ha estado o estudia en colegios privados. Asimismo, el estudio en mención "permitió evidenciar el nivel de frustración, ira, desesperanza, temor y pesimismo que sienten los muchachos y revelan en sus charlas, en sus atuendos, en su lenguaje, en sus acciones e incluso en sus omisiones. La apatía hacia el estudio es uno de los claros síntomas de la pérdida de horizontes en muchachos y muchachas que apenas comienzan su vida".

En relación al capital humano, se ha señalado que Barranquilla presenta una amplia brecha respecto a la cualificación del talento humano representada en las pruebas estandarizadas (SABER, $5^{\circ}, 9^{\circ}, 11^{\circ}$ y últimos semestres de la universidad) e incluso llega a un rezago del $15 \%$ al ser comparada con ciudades tales como Bogotá y Bucaramanga. El autor establece que la principal razón de este hecho, se debe básicamente a las características de los estudiantes al igual que su entorno familiar, de allí que la calidad educativa, su edad y la situación de vinculación laboral por parte de sus padres, evidencian un rendimiento académico deficitario. Bajo esa óptica, se podría afirmar que el progreso académico del estudiante no lo determinan las características de la escuela. En ese orden de ideas, cabe mencionar que Barranquilla y Soledad, tienen aproximadamente un $65 \%$ de trabajadores pobres informales, es decir que además de pobres les compete trabajar de su cuenta y su vinculación a procesos de salud y atención es paupérrima. Sí ha lo postulado anteriormente se le suma que si un individuo se expone 
a contextos donde impera la violencia, el maltrato, el acoso, entre otros, este estado de cosas es el insumo para la reproducción de la violencia.

\section{Metodología}

El presente estudio es de tipo descriptivo-analítico, de corte transversal, realizado en las IED de Barranquilla en el marco del Observatorio Escolar de Convivencia. Establece una muestra de 152 estudiantes e igual número de docentes y directivos de las instituciones educativas oficiales de la ciudad de Barranquilla.

El instrumento empleado es de tipo estructurado y aborda las dimensiones de: Convivencia, Conflicto Escolar e Intervención. Posee tres versiones (El cuestionario dirigido a profesores, el dirigido a directivos y el dirigido a alumnos). Está fundamentado en el "Protocolo para la Medición de la convivencia en los Centro Educativos No universitarios de la Comunidad Autónoma de Aragón" (Gómez-Bahillo, Puyal, Sanz, Elboj, y Sanagustin, 2006) en su respectiva adaptación al contexto de estudio.

\section{Resultados}

Los resultados obtenidos tienen como fuente de confiabilidad el procesamiento en el software estadístico SPSS. En cuanto al análisis de la percepción del ambiente escolar en general, se indican 5 categorías de evaluación: Muy Bueno, Bueno, Normal, Ocasionalmente Mal, y Muy Mal.

Figura 1. Ambiente Escolar

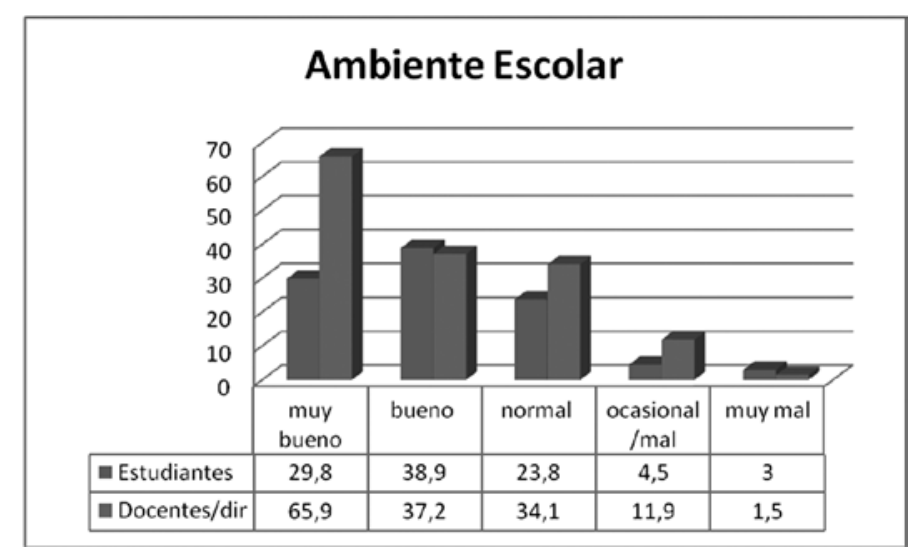

En términos generales, la Figura expresa que tanto estudiantes como docentes y directivos considera que existe en las IED un clima escolar bueno. Se desprende desde la mirada de los actores, que existe una clara diferenciación entre lo que el imaginario estudiantes capta por ambiente escolar y la percepción docente y directiva sobre el impacto de la valoración de dicho criterio y su quehacer como sujeto encargado de direccionar y formar al talento que se encuentra en su centro de formación. Mientras que la mayoría de los docentes y directivos consideran que el clima escolar es muy bueno $(65,9 \%)$, en el caso de los estudiantes la proporción corresponde sólo al 29,8\%.

Existe cierta simetría en torno a la percepción de Bueno; pero al tiempo no alcanza ni un $50 \%$, es decir que tanto estudiantes como docentes y directivos consideran que lo que ocurre como experiencia para ellos, es visto como algo cotidiano y ajustado a lo que el día a día establece al interior del centro, reconociendo que existen anomalías que no permiten la creación de un buen ambiente para la convivencia. Se puede extraer de los datos, que existe un reconocimiento por parte de los actores que esporádicamente se presentan situaciones que evidencian un clima escolar malsano. Este tipo de hechos tiene que ver con: Ofensas verbales, apodos y burlas, agresión física, daño en bien ajeno, hurto y robos entre otros.

En cuanto a las actitudes hacia el centro, analizada sólo en los estudiantes, se estableció una escala de medición de tres valores: Bajo(0-4), Media(5-6) y Alta(7-10). Los resultados indican que el $97.1 \%$ de los estudiantes considera que la motivación a movilizarse hacia la escuela es en términos generales el deseo de aprender.

Figura 2. Deseo de Aprender

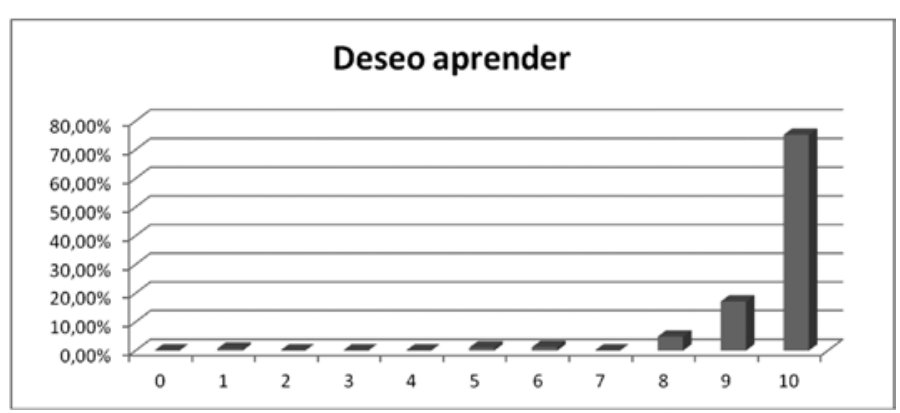


Puede apreciarse que la escuela es un escenario de ascenso, de éxito o fracaso según la relación de empatía entre estudiantes, la misma dinámica que establece la institución de formación, al igual que la demanda de la sociedad. Se ha de considerar la escuela comola segunda red de socialización, de encuentrosy desencuentros; donde además de conocimientos seincorporan manifestaciones socio-afectivas; manifestaciones emocionales extremas y antagónicas, fruto de las declaraciones que los otros hacen de ellos, pero no de un proceso introspectivo.

Considerando la aguda crisis que reflejan las localidades de la ciudad, cobran una importancia crucial las afirmaciones a través de las cuales los estudiantes en su imaginario la conciben, como un escenario de formación para obtener un mejor futuro (86\%).

Figura 3. Visión de Futuro

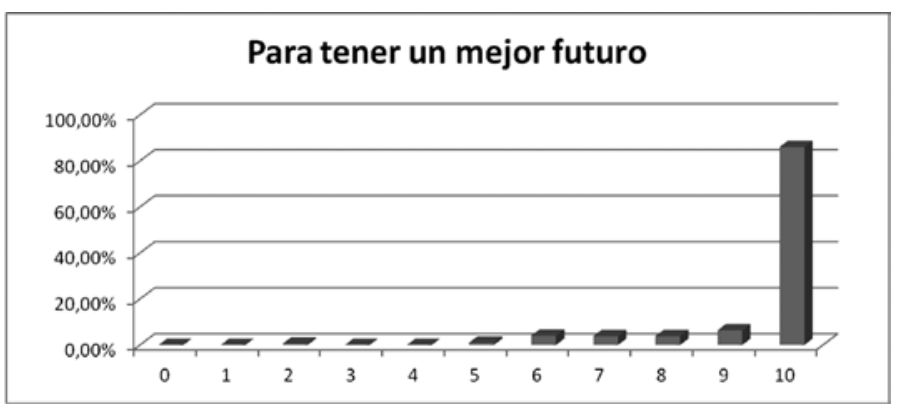

Al mismo tiempo se concibe a la escuela como lugar de interacción, como un espacio para compartir entre pares $(78 \%)$, de allí entonces que la dinámica de la cotidianidad barrial, empiece a ser desplazada para ver en la escuela un escenario que le proporcione tranquilidad y seguridad (77.7\%), al tiempo se considera que lo que representa social y económicamente está respaldado por el deseo de aprender.

Figura 4. Interacción con los amigos

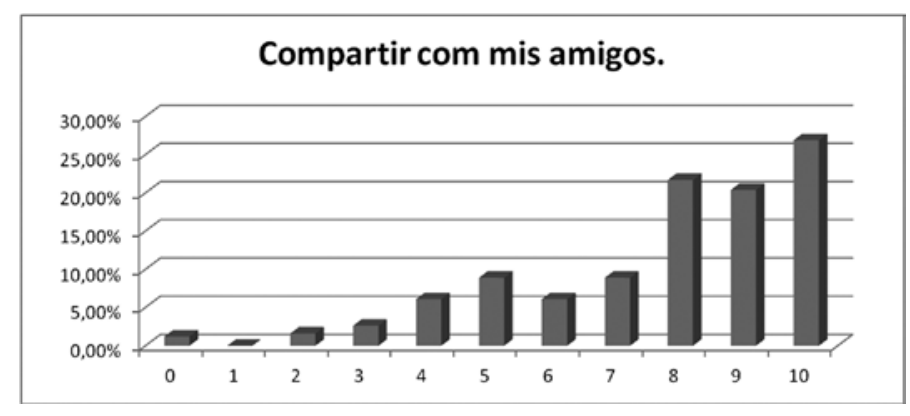

Al mismo tiempo se concibe a la escuela como lugar de interacción, como un espacio para compartir entre pares $(78 \%)$, de allí entonces que la dinámica de la cotidianidad barrial, empiece a ser desplazada para ver en la escuela un escenario que le proporcione tranquilidad y seguridad (77.7\%), al tiempo se considera que lo que representa social y económicamente está respaldado por el deseo de aprender.

Figura 5. Percepción de Tranquilidad y Seguridad

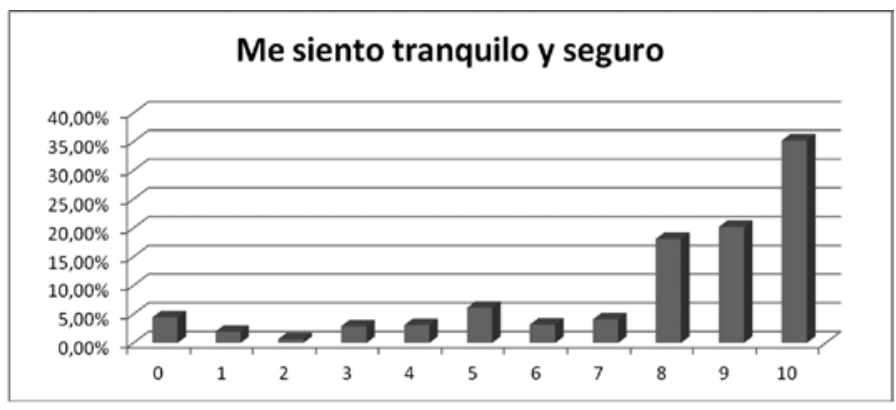

Es de reconocer que existe un $9.8 \%$ de estudiantes que se manifiesta en una frecuencia de bajo en torno a lo que tiene que ver con la seguridad que le brinda la institución; similar situación ocurre cuando un $9.3 \%$ se identifica en medio. Es decir entre bajo y medio nos da un estimativo significativo de $19.1 \%$ que genera una mirada diferente al de la mayoría.

Figura 6. Normatividad de los Padres

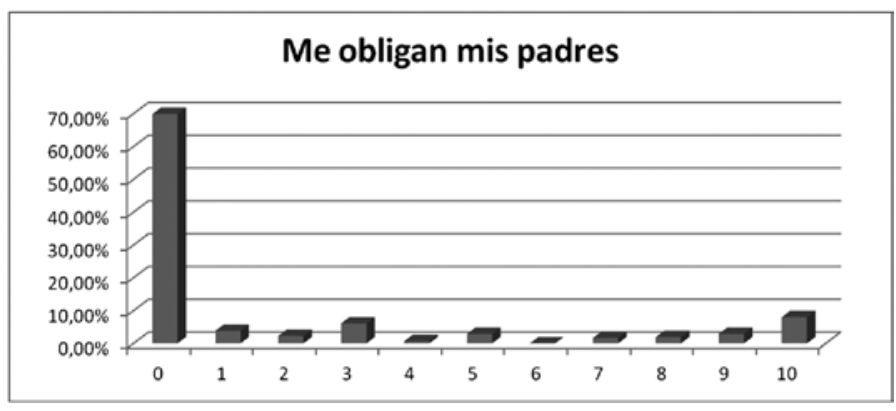

Se reconoce que existen algunas situaciones que generan desfavorabilidad para la consecución de esos grandes ideales que la formación les puede proporcionar. Se percibe en la manifestación del estudiantado que aún persiste la obligatoriedad de los padres para que los hijos acudan al centro de formación (14.5\%), pero también se genera una apreciación que amerita un mayor 
análisis como es el hecho de acudir a la escuela para "olvidar los problemas de casa"; es decir la escuela se convierte en un escenario que posibilita una estructura de beneficios que en casa no encuentra.

Lo paradójico es que en los procesos de intervención para la formación de ese talento humano, se desconoce total o parcialmente los anhelos e interés del estudiantado; máxime desde los procesos de evaluación y de empatía en la relación docente-estudiante, se desconoce como el maltrato, la agresión entre pares es causal de deserción, por lo cual empírica y asistencialmente se han planteado estrategias para mitigar los conflictos y fortalecer una mejor convivencia, sin embargo los mismos son de corta duración y sin trascendencia, cayendo en un activismo ante los organismos de control Distrital.

Figura 7. Evitación de problemas en el hogar

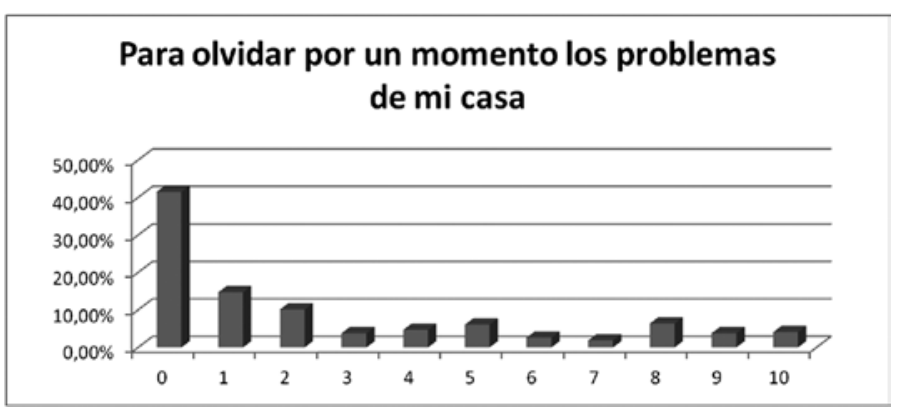

Percepción de las interacciones entre pares estudiantiles.

Figura 8. Relaciones Interpersonales Agradables

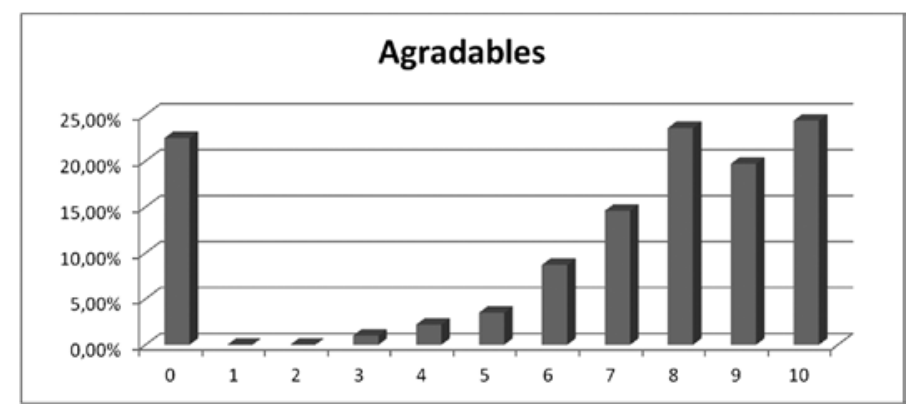

El tercer factor de análisis de la presente investigación, se orienta a las relaciones interpersonales entre los pares escolares. También se estable el grupo de criterios sobre favorabilidad y desfavorabilidad; con una frecuencia entre Bajo, Media y Alta.

Los resultados obtenidos ofrecen una mirada tanto de estudiantes como de docentes, en la que se señala que las relaciones interpersonales se caracterizan por los altos niveles de agradabilidad, confianza, alta dosis de afecto y persistencia de una cuota de tolerancia. Sin embargo en las relaciones interpersonales, emergen una serie de connotaciones que son las que conducen a las diversas expresiones del conflicto escolar; siendo el irrespeto, el enfrentamiento entre pares, el egoísmo, las expresiones del clima escolar imperante en la ciudad de Barranquilla y las mismas adquieren diversas manifestaciones: las ofensas verbales, los apodos y burlas, las agresiones físicas, el daño en bien ajeno, el hurto, la extorsión, la amenaza, hacen parte de las variables que hoy transitan en los centros educativos como dispositivos que afectan la sana convivencia.

Figura 9. Tolerancia en las Relaciones Interpersonales

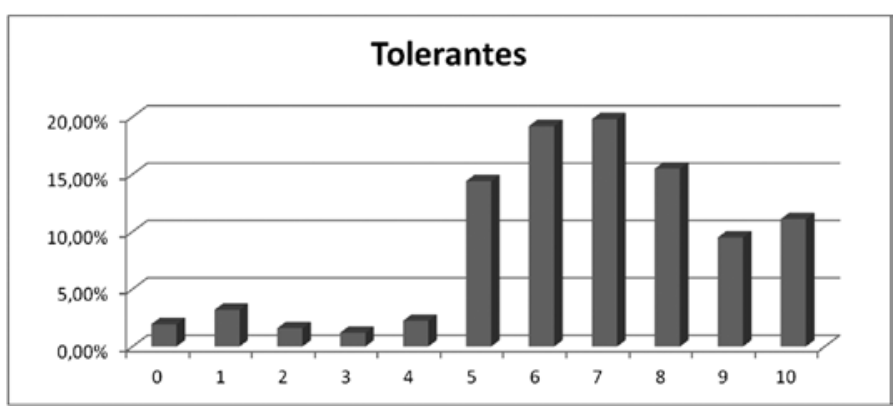

Figura 10. Confianza en las Relaciones Interpersonales

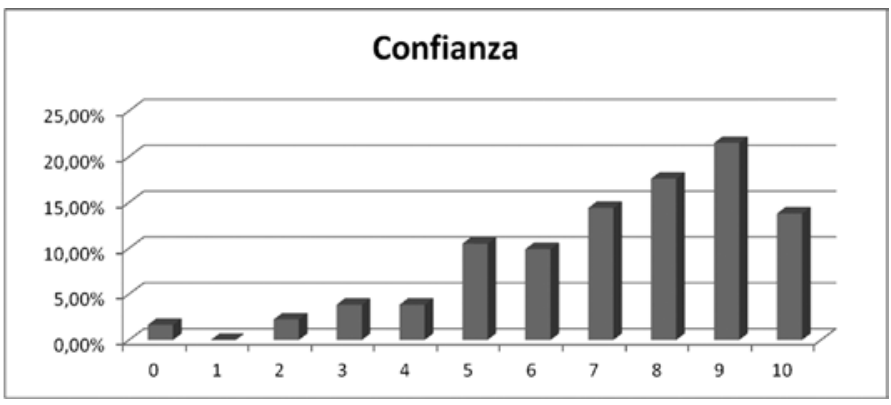


Figura 11. Expresiones de Afecto en las Relaciones Interpersonales

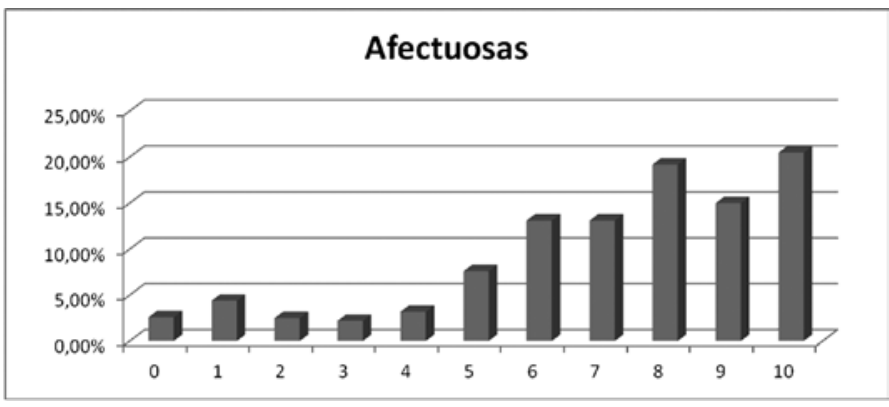

Figura 12. Percepción del Egoísmo

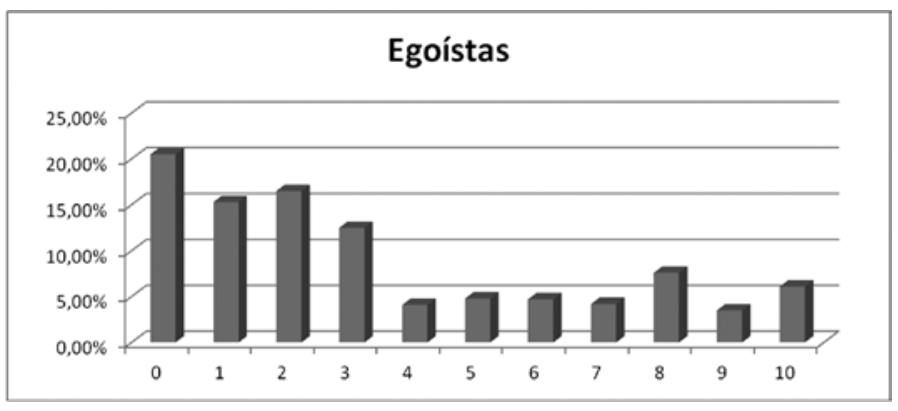

Figura 13. Situaciones Interpersonales Conflictivas

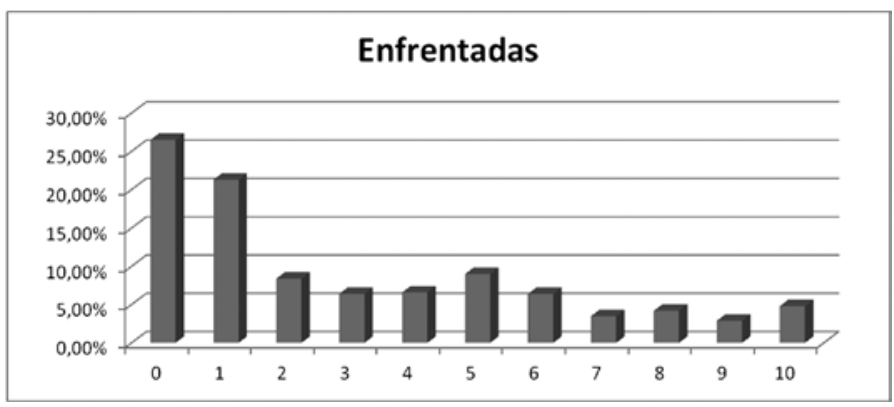

\section{Discusión}

Los resultados obtenidos permiten identificar que existe un clima escolar nutritivo entre los estudiantes, docentes y directivos de las IED de Barranquilla; sin embargo, emerge en la interacción de pares escolares manifestaciones de agresividad la cual se constituye en un estado emocional que se asocia a una diversidad de distorsiones en el individuo, que pueden ir desde factores cognitivos, motrices y verbales; la agresividad ha de entenderse entonces como una experiencia interna y no tiene necesariamente que conducir a la agresión; sin embargo hay que considerar las diversas respuestas que internamente experimenta el sujeto y luego es esa manifestación externa la que "nos empuja a agredir".

Los datos del Observatorio de Convivencia de la ciudad de Barranquilla, nos invitan a indagar sobre el tipo de agresión imperante según su función, pues al cruzar la información de las baterías como es el caso de las de intervención psicosocial donde hay un reconocimiento expreso del desarrollo de riñas en el contexto barrial, a su vez existe presencia de actores involucrados en este tipo de hechos en los centros educativos, el desarrollo de estas acciones declina sin que ello quiera decir que desaparecen; por ello, se evidencia en la muestra, dos tipos de agresión.

Desde los aportes de Chaux (2012) en primera instancia sobre la Agresión reactiva que se refiere al uso de la agresión como respuesta ante una ofensa real o percibida. Es el insulto o el golpe con el que responde alguien cuando siente que otra persona lo ha herido. En segunda instancia frente a la agresión instrumental (también conocida como agresión proactiva), en cambio, no está precedida de ninguna ofensa.

Los hallazgos a su vez generan reflexiones complementarias según las cuales es importante preguntarse si uso de la agresión puede ser concebido como un instrumento para conseguir un objetivo, sea éste recursos, dominación, estatus social o algo más". Sí este tipo de manifestación impera en las aulas Distritales, ¿Están las escuelas y docentes preparados para manejar competencias de tipo afectivo e intencionado con ese tipo de población?, ¿De qué manera vincular a las familias para el manejo asertivo de este tipo de competencias?, ¿Cómo evaluar el impacto de la incorporación afectiva en el aula?.

No hay que desconocer bajo el panorama descrito anteriormente, que en las relaciones interpersonales, el conflicto escolar está presente; pero este, es de carácter estructural y se a trasladó a la escuela; es necesario, por tanto, que ella interpele positivamente la dinámica de la ciudad. En ese orden de ideas, surge una serie de factores asociados al conflicto en las instituciones 
educativas, que si bien no son determinantes, están incidiendo en el comportamiento de los actores inmediatos: los estudiantes.

El contexto o perímetro donde se encuentran las instituciones educativas se mueven en la cotidianidad del consumo de alcohol, altas tasas de desempleo, la delincuencia, la recurrencia de la drogadicción, la emergencia del microtráfico que se refleja al interior del centro por medio del hurto como forma de subsistencia, y la organización de pandillas juveniles como formas de visibilizar "poder" ante sus pares y al mismo centro de formación; sin desconocer una variable que arroja el observatorio, como es la desnutrición que se asocia a la línea de pobreza, como factor de escalada en el conflicto escolar. Estos resultados se articulan funcionalmente con los aportes de García-Pujadas, Pérez-Almaguer, y Hernández-Batista, R. (2013) en su estudio sobre los factores asociados con la convivencia escolar en el nivel secundario y en particular frente al rol de los adolescentes y sus modelos de interacción social escolar.

En ese orden de ideas, puede apreciarse que al interior de las escuelas existen algunos lugares donde se desarrolla el conflicto escolar: el salón de clases, para posteriormente, señalar que también se registra este fenómeno en las afueras del colegio. Lo anterior supone en primera instancia que algunos hechos de disrupción sean catalogados como manifestación del conflicto escolar en el caso del aula, y en segunda instancia, la elaboración de mutuos acuerdos para el desarrollo de la manifestación de la violencia por fuera del colegio ante las disposiciones disciplinarias que este hecho podría generar. Obsérvese que este planteamiento, aplica para los escenarios deportivos. Igualmente, llama la atención que las redes sociales son también infraestructura para el desarrollo de los conflictos escolares; sin embargo, esta en los dos años ha mantenido su tendencia baja.

Es decir, la web sigue utilizándose como instancia para el conflicto, mediando a través de esta los apodos y las burlas. Es de considerar que dichas manifestaciones de conflicto e incluso violencia entre pares escolares se generan según el objeto y alcance de la investigación en estudiantes que están entre 13 a 15 años, incluso se observa que hay un incremento de conflictos entre pares de 16 a 18 años.

De lo anterior se desprende que el primer grupo absorbe el conjunto de estereotipos que la sociedad en su generalidad expone, máxime en estos dos últimos años, cuando en los medios masivos de comunicación se hace apología al delito, al uso de las acciones violentas para darle solución a un problema, las manifestaciones de ofensas verbales entre mandatarios, la polarización de la misma sociedad, entre otras. Estos hallazgos se relacionan con los aportes de García \& Cubo (2009) quienes formulan un plan acciones estratégicas para el mejoramiento del clima escolar.

En dichas interacciones conducentes al conflicto escolar, se identifica el rol de los actores: víctima, victimario y observadores; de donde se aprecia un aumento de los victimarios en detrimento de sus víctimas y un grupo significativos de observadores que se masifican cuando a la víctima se le acosa. Esta última en una asimetría de poder sufre física y psicológicamente por el cúmulo de agresiones recibidas.

En este sentido Kröyer, Muñoz \& Ansorena (2012) recomiendan el desarrollo de modelos normativos ajustados al contexto escolar que permitan generar un espacio prosocial y constructivo más que restrictivo.

En términos generales, el análisis de los datos de la presente investigación permite continuar investigando acerca del fenómeno del "Clima escolar" y su repercusión en la convivencia, puesto que a pesar de la buena percepción que poseen los estudiantes, directivos y docentes, en la práctica académica aún se observan manifestaciones de agresión, matoneo, bullying, ciberbullying, fracaso y deserción escolar, que reflejan un incremento en los titulares de la prensa y en la estadística de casos reportados. Estos últimos aspectos relacionados con el matoneo han sido ampliamente analizados por Hoyos (2005) entre otros.

Finalmente y en consistencia con los estudios de Rodríguez \& Vaca (2010), surge la propuesta de desarrollar procesos comunitarios permanentes de promoción de 
la convivencia escolar, que a su vez permitan el monitoreo y seguimiento continuo de los procesos que se adelantan en el Observatorio de Convivencia Escolar.

\section{Referencias}

Arón, A. \& Milicic, N. (1999). Clima social escolar y desarrollo personal: Un programa de mejoramiento. Editorial Andrés Bello: Santiago de Chile.

Arón, A.M. \& Milicic, N. (2000). Climas Sociales Tóxicos y Climas Nutritivos para el Desarrollo Personal en el Contexto Escolar. Revista Psykhé, 9 (1), 117-124.

Ascorra, A., Arias, H. \& Graff, C. (2003). La escuela como contexto de contención social y afectiva. Revista Enfoques Educacionales, 5 (1), 117-123.

Berkowitz, L. (1996). Agresión. Causas, consecuencias y control. Bilbao: DDB.

Blaya, C., Debarbieux, E., Rey-Alamillo, R. \& OrtegaRuiz, R. (2006). Clima y violencia escolar. Un estudio comparativo entre España y Francia. Revista de Educación, 339 (1) 293-331.

Cere. (1993). Evaluar el contexto educativo. Documento de Estudio. Vitoria: Ministerio de Educación y Cultura, Gobierno Vasco.

Cepeda, L. (2013). La economía de Barranquilla a comienzos del sigloXXI. Bogotá: Banco de la República.

Chaux, E. (2010). Aulas en Paz: Alternativa a la violencia escolar. Periódico de la Policía Nacional de Colombia, 4, 15.

Chaux, E. (2012). Educación, convivencia y agresión escolar. Bogotá: Ediciones Uniandes. Taurus, Santillana.

Cornejo, R y Redondo, J. (2001). El clima escolar percibido por los alumnos de enseñanza media. Una investigación en algunos liceos de la región metropolitana. Última Década, 15, 11-52.

Erazo-Santander, O. A. (2010). Reflexiones sobre la violencia escolar. Revista de Psicología GEPU, 1 (3), $74-86$.

Forero-Londoño, O. F. (2011). La violencia escolar como régimen de visibilidad. Magis, Revista Internacional de Investigación en Educación, Julio-Diciembre, 399-413.

García-H. M. \& Cubo D. S. (2009). Convivencia escolar en Secundaria: aplicación de un modelo de mejora del clima social. REIFOP, 12 (1), 51-62. Recuperado de: http://www.aufop.com/
García-Pujadas, M. I., Pérez-Almaguer, R.y HernándezBatista, R. (2013) Convivencia escolar en secundaria básica. Ciencias Holguín, 19 (3), julio-septiembre, 1-11. Recuperado de: http://www.redalyc.org/ pdf/1815/181528709007.pdf

Gómez-Bahillo, C., Puyal, E., Sanz, A., Elboj, C. y Sanagustin, M. (2006). Las relaciones de convivencia y conflicto escolar en los centros educativos aragoneses de enseñanza no Universitaria. Análisis y propuesta desde una perspectiva socioeducativa. España: Gobierno de Aragón.

Hermida, M., Valencia, I. y Madariaga, C. (2013). Intervención psicosocial a jóvenes en situación de riesgo pertenecientes a pandillas en el Distrito de Barranquilla. Colombia: Alcaldía Distrital de Barranquilla.

Hoy. W. K. y Miskel, C. G. (1996). Educational administration. Theory, research and practice ( $\left.5^{\mathrm{a}} \mathrm{ed}.\right)$. Londres: McGraw-Hill.

Hoyos, O. (2005). Caracterización del maltrato entre iguales en una muestra de colegios de Barranquilla (Colombia). Psicología desde el Caribe, 16 (1), 1-28, 2005.

Hoyos, O., Romero, L., Valega, S. y Molinares, C. (2009). El maltrato entre iguales por abuso de poder y exclusión social en estudiantes de una universidad privada de la ciudad de Barranquilla. Pensamiento Psicológico, 6 (13), 109-125.

Hoyos, O. L. R., Llanos M. M. \& Valega, S. J. (2012). El maltrato entre iguales por abuso de poder en el contexto universitario: incidencia, manifestaciones y estrategias de solución. Universitas Psychologica, 11 (3), 793-802.

Kröyer, O. N., Muñoz Reyes, M. \& Ansorena Carrasco, N. E. (2012). Normativa y reglamentos de convivencia escolar, ¿una oportunidad o una carga para la escuela?. Educere, 16(55), 373-384. Recuperado de: http://www.redalyc.org/articulo. oa?id=35626140002

Lacouture, G. (1996) El legado de Kurt Lewin. Revista Latinoamericana de Psicología, 28 (1), 159 -163. Recuperado de: http://www.redalyc.org/ pdf/805/80528113.pdf

Peñaranda, F. (2006). Hacia una comprensión de la construcción de significados sobre la crianza en el programa de crecimiento. Manizales - Colombia. Tesis doctoral, Cinde - Universidad de Manizales. 
Plan Distrital de Educación 2010-2020. Secretaría de Educación de Barranquilla.

Ministerio de Educación Nacional (2005) Plan Nacional Decenal de Educación 2006-2016.

Rodríguez, D. (2004). Diagnóstico organizacional. Santiago: Ediciones Universidad Católica de Chile.

Rodríguez-Jarez, X. (2004). Educar para la paz en tiempos difíciles. España: Bakeaz.

Rodríguez, M. C. \& Vaca P. (2010) Promover la convivencia escolar: una propuesta de intervención comunitaria. Aletheia, 33(3), 179-189. Recuperado de: http:// www.redalyc.org/pdf/1150/115021494016.pdf
Torres, L. y Páez, K. (2010). Caracterización de los Conflictos Escolares en los grados Octavo y Noveno, en instituciones de Barranquilla. Tesis de Maestría no publicada. SUE-Caribe. Universidad del Atlántico. Barranquilla, Colombia.

Valdés, H., Treviño, E., Castro, M., Carrillo, S., Bogoya, D., Costilla, R. y Acevedo, G. (2008). Los aprendizajes de los estudiantes de América Latina y el Caribe. Primer reporte de los resultados del Segundo Estudio Regional comparativo y Explicativo, Santiago de Chile, Oficina Regional de Educación dela UNESCO para América Latina y el Caribe. 